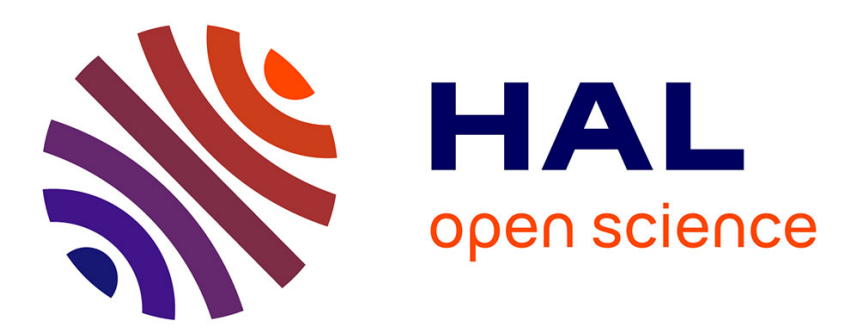

\title{
Optimal instrumental variable method for closed-loop identification
}

Marion Gilson, Hugues Garnier, Peter J.W. Young, Paul van den Hof

\section{To cite this version:}

Marion Gilson, Hugues Garnier, Peter J.W. Young, Paul van den Hof. Optimal instrumental variable method for closed-loop identification. IET Control Theory and Applications, 2011, 5 (10), pp.11471154. 10.1049/iet-cta.2009.0476 . hal-00594388

\section{HAL Id: hal-00594388 \\ https://hal.science/hal-00594388}

Submitted on 19 May 2011

HAL is a multi-disciplinary open access archive for the deposit and dissemination of scientific research documents, whether they are published or not. The documents may come from teaching and research institutions in France or abroad, or from public or private research centers.
L'archive ouverte pluridisciplinaire HAL, est destinée au dépôt et à la diffusion de documents scientifiques de niveau recherche, publiés ou non, émanant des établissements d'enseignement et de recherche français ou étrangers, des laboratoires publics ou privés. 


\title{
Optimal instrumental variable method for closed-loop identification*
}

\author{
Marion Gilson*†, Hugues Garnier*, \\ Peter C. Young**, Paul M.J. Van den Hof***
}

*Centre de Recherche en Automatique de Nancy, Nancy-Université, CNRS, BP 70239, F-54506 Vandœuvre-les-Nancy, France.

[marion.gilson, hugues.garnier] @cran.uhp-nancy.fr

**Centre for Research on Environmental Systems and Statistics,

Systems and Control Group, Lancaster Environment Centre, Lancaster, LA1 4YQ, UK

Fenner School of Environment and Society, Australian National University, Canberra, Australia

School of Electrical Engineering and Telecommunications, University of New South Wales, Sydney, Australia.

p.young@lancaster.ac.uk

***Delft Center for Systems and Control, Delft University of Technology,

Mekelweg 2, 2628 CD Delft, The Netherlands.

p.m.j.vandenhof@tudelft.nl

\begin{abstract}
This paper presents in a new unified way, optimal instrumental variable methods for identifying discrete-time transfer function models when the system operates in closed-loop. The conditions for the optimal design of prefilters and instruments depending on common model structures are analyzed and different approaches are developed according to whether the controller is known or not. The performance of the proposed approaches is evaluated by Monte-Carlo analysis in comparison with other alternative closed-loop estimation methods.
\end{abstract}

Key words: System identification; closed-loop identification; optimal instrumental variable; Box-Jenkins models.

\footnotetext{
*Paper submitted for possible publication in IET Control Theory and Applications
}

${ }^{\dagger}$ Corresponding author. 


\section{Introduction}

Feedback is present in a variety of practical situations due to safety and/or economic restrictions. In the last two decades, various attempts have been made to handle linear system identification in the presence of feedback. The main difficulties are well-known and due to the correlation between the disturbances and the control signal induced by the loop. Identification methods dealing with closed-loop experimental data were first developed in the eighties ([18], [12]); several overviews gather these results (see e.g. [2], [20], [1]). Renewed attention has been given lately to the problem of closed-loop identification initiated by an emerging interest in identification for model-based control ([3], [10], [7]) and/or experiment design in the closed-loop framework $([15],[8])$. Several methods have been developed recently, therefore, and may be divided into two main approaches depending on the chosen state-space (see e.g. [9] for a survey) or transfer function model form. In this paper, transfer function model identification via instrumental variable (IV) methods is considered.

The IV technique to the identification of transfer function models has indeed a rich history in the control and system literature, with the earliest algorithms of this type dating back to the 1970s for the open-loop case [22] and 1980s for the closed-loop situation [19]. More recently, a so-called 'tailor-made IV algorithm' was proposed [5] where the closed-loop plant is parameterized using the (open-loop) plant parameters. A connexion with the bias eliminated least-squares method was also discussed $([28],[27])$. Then, an optimal (minimal) variance result was developed in the closed-loop extended IV identification case, revealing consequences for the choice of weights, filters and instruments. Two bootstrap techniques were developed for estimating AR and ARARX models [6].

Attention has been given very recently to more general models and optimal IV solutions are presented either for linear model identification (see e.g. [16] or [4] for Box-Jenkins hybrid continuous-time models) or non-linear models [11]. This paper outlines the main aspects of a statistically optimal refined IV approach for the identification of common use structures as ARX, ARARX, OE and BJ, in closed loop. The BJ model structure presents the advantage of allowing the plant and the noise models to have different dynamics and it can be shown that the estimates of the noise model parameters are asymptotically independent of the process model parameter estimates (see [24] and the prior references therein). An apparent problem with this type of BJ model is that simple IV estimation cannot be used directly because the model is nonlinear-in-the-parameters. However, more sophisticated IV estimation can be used to overcome this limitation [24]. Indeed, the identification problem is rewritten in this paper to make use of a linear-in-the-parameters predictor next to an additional noise model identification required for determining the optimal prefilter and instrument.

This paper provides, therefore, a survey on the statistically optimal IV solutions for the estimation of discrete-time transfer function models when the system operates in closed-loop, and 
whatever the controller knowledge is. In this regard, it can be compared to the well-known Prediction Error Minimization (PEM) approach [12]. However, as illustrated in this paper, the proposed refined IV algorithm seems, as in the open-loop case [24], less sensitive than the PEM algorithm to the initialization [14].

The paper is organized as follows. After the preliminaries, the optimal IV solution is presented according to the model structure used, and the controller knowledge. Finally, the different methods are compared with the help of Monte-Carlo simulation examples.

\section{Problem formulation}

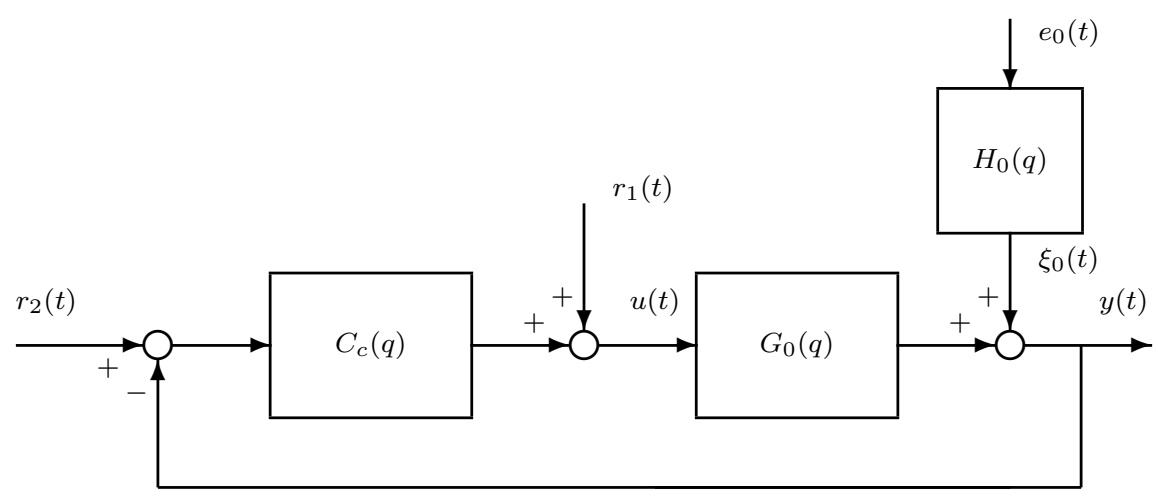

Figure 1: Closed-loop system configuration

\section{Preliminaries}

Consider a stable, linear, Single Input Single Output (SISO), closed-loop system of the form shown in Figure 1. The data generating system is assumed to be given by the following relations

$$
\mathcal{S}:\left\{\begin{array}{l}
y(t)=G_{0}(q) u(t)+H_{0}(q) e_{0}(t) \\
u(t)=r(t)-C_{c}(q) y(t), \text { where } r(t)=r_{1}(t)+C_{c}(q) r_{2}(t) .
\end{array}\right.
$$

The plant is denoted by $G_{0}(q)=B_{0}\left(q^{-1}\right) / F_{0}\left(q^{-1}\right)$ with the numerator and denominator degree equals to $n_{0}$, the controller is denoted by $C_{c}(q)$ and $q^{-1}$ is the delay operator with $q^{-i} x(t)=$ $x(t-i) . u(t)$ describes the plant input signal, $y(t)$ the plant output signal. A coloured disturbance $\xi_{0}(t)=H_{0}(q) e_{0}(t)$ is assumed to affect the closed-loop, where $e_{0}(t)$ is a white noise, with zero mean and variance $\sigma_{e_{0}}^{2}$.

The following general model structure is chosen to model the system (1)

$$
\mathcal{M}: y(t)=G(q, \rho) u(t)+H(q, \eta) \varepsilon(t, \theta),
$$

where the parameter vector is given as $\theta^{T}=\left(\rho^{T} \eta^{T}\right)$. The parameterized plant model takes then the form,

$$
\mathcal{G}: G(q, \rho)=\frac{B\left(q^{-1}, \rho\right)}{F\left(q^{-1}, \rho\right)}=\frac{b_{1} q^{-1}+\cdots+b_{n} q^{-n}}{1+f_{1} q^{-1}+\cdots+f_{n} q^{-n}},
$$


where $n$ denotes the plant model order and with the pair $(F, B)$ assumed to be coprime. The plant model parameters are stacked columnwise in the parameter vector

$$
\rho=\left[\begin{array}{llllll}
f_{1} & \cdots & f_{n} & b_{1} & \cdots & b_{n}
\end{array}\right]^{T} \in \mathbb{R}^{2 n} .
$$

The plant model order $n$ is assumed known or identified from the data and the parameterized noise model is assumed to be in the form of the following ARMA process,

$$
\mathcal{H}: H(q, \eta)=\frac{C\left(q^{-1}, \eta\right)}{D\left(q^{-1}, \eta\right)}=\frac{1+c_{1} q^{-1}+\cdots+c_{m} q^{-m}}{1+d_{1} q^{-1}+\cdots+d_{m} q^{-m}},
$$

where the associated noise model parameters are stacked columnwise in the parameter vector,

$$
\eta=\left[\begin{array}{llllll}
d_{1} & \cdots & d_{m} & c_{1} & \cdots & c_{m}
\end{array}\right]^{T} \in \mathbb{R}^{2 m} .
$$

Note that this paper deals with IV-based methods which are known to give consistent plant model parameter estimates of $G_{0}$ irrespective of the structure of $H_{0}$.

Now consider the relationship between the plant input and output signals in (1),

$$
y(t)=G_{0}(q) u(t)+H_{0}(q) e_{0}(t) .
$$

If the plant $G_{0}$ is included into the chosen model set $\mathcal{G}\left(G_{0} \in \mathcal{G}\right), y(t)$ can be written as

$$
y(t)=\varphi^{T}(t) \rho_{0}+v_{0}(t)
$$

where $\rho_{0}$ denotes the true plant parameter vector,

$$
\varphi^{T}(t)=[-y(t-1) \cdots-y(t-n) u(t-1) \cdots u(t-n)]
$$

and $v_{0}(t)=F_{0}\left(q^{-1}\right) H_{0}(q) e_{0}(t)$.

In the following, the closed-loop system is assumed to be asymptotically stable and $r(t)$ is an external signal that is persistently exciting of sufficient high order.

The objective is then to estimate the parameter vector from the collected sampled data $y(t)$, $u(t)$ and $r(t)$ when the controller $C_{c}(q)$ is known or not.

Let us introduce some notations used in the following Sections. The closed-loop system can be split up into two different parts as follows

$$
\begin{aligned}
& y(t)=\frac{G_{0}(q)}{1+C_{c}(q) G_{0}(q)} r(t)+\frac{1}{1+C_{c}(q) G_{0}(q)} \xi_{o}(t)=\grave{y}(t)+\tilde{y}(t) \\
& u(t)=\frac{1}{1+C_{c}(q) G_{0}(q)} r(t)-\frac{C_{c}(q)}{1+C_{c}(q) G_{0}(q)} \xi_{o}(t)=\stackrel{u}{u}(t)+\tilde{u}(t),
\end{aligned}
$$

where $\stackrel{y}{y}(t)$ and $\dot{u}(t)$ are the noise-free input/output, $\tilde{y}(t)$ and $\tilde{u}(t)$ the noise part of $y(t)$ and $u(t)$ respectively. The noise-free regressor $\dot{\varphi}^{T}(t)$ is then defined on the basis of these signals as follows

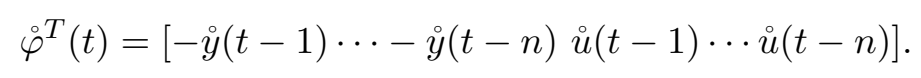




\section{IV method for closed-loop identification}

\subsection{Extended IV}

The extended-IV estimate is given by (see e.g. [17])

$$
\hat{\rho}_{x i v}(N)=\arg \min _{\rho}\left\|\left[\frac{1}{N} \sum_{t=1}^{N} L(q) \zeta(t) L(q) \varphi^{T}(t)\right] \rho-\left[\frac{1}{N} \sum_{t=1}^{N} L(q) \zeta(t) L(q) y(t)\right]\right\|_{W}^{2},
$$

where $\zeta(t) \in \mathbb{R}^{n_{\zeta}}$ with $n_{\zeta} \geq 2 n$ is the instrument vector, $\|x\|_{W}^{2}=x^{T} W x$, with $W$ a positive definite weighting matrix and $L(q)$ a stable prefilter.

By definition, when $G_{0} \in \mathcal{G}$, the extended-IV estimate is consistent under the following two conditions $^{1}$

- $\overline{\mathbb{E}} L(q) \zeta(t) L(q) \varphi^{T}(t)$ is full column rank,

- $\overline{\mathbb{E}} L(q) \zeta(t) L(q) v_{0}(t)=0$.

\subsection{Lower bound for an IV method}

The choice of the instrumental variable vector $\zeta(t)$, the number of instruments $n_{\zeta}$, the weighting matrix $W$ and the prefilter $L(q)$ may have a considerable effect on the covariance matrix $P_{x i v}$ produced by the IV estimation algorithm. In the open-loop situation the lower bound of the covariance matrix for any unbiased identification method is given by the Cramer-Rao bound (see e.g. [17] and [12]). The closed-loop situation has been investigated more recently in [6]. The main results to be used in the following are only briefly recalled here. It was shown that a minimum value of the covariance matrix $P_{x i v}$ as a function of the design variables $\zeta(t), L(q)$ and $W$ exists under the restriction that $\zeta(t)$ is a causal function of the external signal $r(t)$ only. In that case $P_{x i v} \geq P_{x i v}^{o p t}$ with

$$
\begin{aligned}
P_{x i v}^{o p t} & =\sigma_{e_{0}}^{2}\left[\overline{\mathbb{E}} \dot{\varphi}_{f}(t) \stackrel{\circ}{f}_{f}^{T}(t)\right]^{-1}, \\
\dot{\varphi}_{f}(t) & =L^{o p t}(q) \stackrel{\varphi}{(}(t), \\
L^{o p t}(q) & =\frac{1}{F_{0}\left(q^{-1}\right) H_{0}(q)}, \text { and } \zeta(t)=\grave{\varphi}(t) .
\end{aligned}
$$

where $\dot{\varphi}(t)$ is the noise-free part of $\varphi(t)$ (see Section 2). Using equations (12) and (13)-(15), the following IV estimate is optimal

$$
\hat{\rho}^{o p t}(N)=\left(\sum_{t=1}^{N} \zeta_{f}(t) \varphi_{f}^{T}(t)\right)^{-1}\left(\sum_{t=1}^{N} \zeta_{f}(t) y_{f}(t)\right)
$$

and where the regressor $\varphi_{f}(t)=L^{o p t}(q) \varphi(t)$, the output $y_{f}(t)=L^{o p t}(q) y(t)$ and the instrument vector $\zeta_{f}(t)=L^{o p t}(q) \zeta(t)$ are filtered by $L^{o p t}(q)(15)$.

\footnotetext{
${ }^{1}$ The notation $\overline{\mathbb{E}}[]=.\lim _{N \rightarrow \infty} \frac{1}{N} \sum_{t=1}^{N} \mathbb{E}[$.$] is adopted from the prediction error framework of [12].$
} 
It has to be noted that in the IV estimator considered in (16), the optimal choice of instruments and prefilter is dependent on unknown system properties, i.e. the plant as well as the noise dynamics. Whereas dependency of plant dynamics could be taken care of by an iterative procedure where the instrument and prefilter are constructed on the basis of a previous plant model estimate $\hat{\rho}^{i-1}$. Knowledge of the noise dynamics is generally missing in an extended IV estimator like (12) as it is not particularly estimated.

Therefore the next step to an optimal IV method should be to extend the estimator (12) with a procedure to estimate an appropriate noise model, to be used as a basis for constructing the optimal prefilter $L^{\text {opt }}(q)$ given in (15).

\section{Optimal IV identification in closed-loop}

\subsection{IV solutions for commonly used model structures in closed-loop}

Optimal IV identification is based on (12). However, it requires the knowledge or the estimation of the noise model to build the filter. In this perspective, a general prediction error identification step is added to estimate a model for $H_{0}(q)$. This requires the choice of a particular parametrization for $H(q, \theta)$, possibly in relation to $G(q, \rho)$.

Two common types of model structure ${ }^{2}$ have been used so far in the literature [6].

- ARX model structure:

$$
A\left(q^{-1}, \rho\right) y(t)=B\left(q^{-1}, \rho\right) u(t)+\varepsilon(t, \rho) .
$$

In this case the noise model

$$
H(q, \hat{\rho})=\frac{1}{A\left(q^{-1}, \hat{\rho}\right)}
$$

is already available from the plant model estimate, and no additional noise model estimate is required. On the basis of the estimated plant model $G(q, \hat{\rho})$ the corresponding filter is given by

$$
L(q, \hat{\rho})=\frac{1}{A\left(q^{-1}, \hat{\rho}\right) H(q, \hat{\rho})}=1
$$

$\hat{\rho}$ is estimated during the plant model identification step.

- ARARX model structure:

$$
A\left(q^{-1}, \rho\right) y(t)=B\left(q^{-1}, \rho\right) u(t)+\frac{1}{D\left(q^{-1}, \eta\right)} \varepsilon(t, \theta) .
$$

In this case the corresponding noise model

$$
H(q, \eta)=\frac{1}{A\left(q^{-1}, \hat{\rho}\right) D\left(q^{-1}, \eta\right)}
$$

\footnotetext{
${ }^{2}$ According to standard PE identification literature the autoregressive part of the following models is denoted by polynomial $A$ (in contrast with the notation $F$ in section 2 ).
} 
is not available from the plant model $\hat{\rho}$ only as previously, but an additional noise model estimate is required. In [6], this is achieved by identifying $D\left(q^{-1}, \eta\right)$ as an autoregressive model $D\left(q^{-1}, \eta\right) w(t)=\varepsilon(t, \eta)$ with

$$
w(t)=A\left(q^{-1}, \hat{\rho}\right) y(t)-B\left(q^{-1}, \hat{\rho}\right) u(t),
$$

by using a first plant model estimate $\hat{\rho}$ and by applying a least square estimator

$$
\hat{\eta}=\arg \min _{\eta} \frac{1}{N} \sum_{t=1}^{N} \varepsilon(t, \eta)^{2} .
$$

The optimal filter that results from this procedure is therefore

$$
L(q, \hat{\eta})=D\left(q^{-1}, \hat{\eta}\right)
$$

Note that these two methods rely on special structures of the assumed noise models, and therefore limit the possibility to reach consistent estimates of $H_{0}(q)$ in situations where these structures are not in accordance with the properties of the underlying systems. Therefore, the IV estimators are then extended to other more general $\mathrm{OE}$ and BJ model structures.

- BJ model structure:

The more general structure is obtained by choosing a Box-Jenkins (BJ) model

$$
y(t)=\frac{B\left(q^{-1}, \rho\right)}{F\left(q^{-1}, \rho\right)} u(t)+\frac{C\left(q^{-1}, \eta\right)}{D\left(q^{-1}, \eta\right)} \varepsilon(t, \rho, \eta),
$$

The IV estimator (16) cannot be directly applied to this non-linear-in-the-parameter model structure (as in [6]). Therefore, the solution is to deal with an IV estimator for the process model coupled with a noise model estimation. This approach is stimulated by the previous development of the refined IV method for open-loop system identification (see [23], [25], [26] and [24] for a unified form that deals with both discrete and continuous-time models).

A natural way to extend the IV estimator (12) with an estimation of the noise model $\eta$ is to write

$$
v(t)=\frac{C\left(q^{-1}, \eta\right)}{D\left(q^{-1}, \eta\right)} \varepsilon(t, \hat{\rho}, \eta)
$$

with $v(t)=y(t)-B\left(q^{-1}, \hat{\rho}\right) / F\left(q^{-1}, \hat{\rho}\right) u(t)$ being available as a measured/reconstructed signal once IV estimator (12) has delivered a plant model $\hat{\rho}$ (see the algorithm Section 4.3). Estimation of $\eta$ in the above equation is then undertaken by an ARMA estimation algorithm on the basis of $v(t)$.

The optimal prefilter that results from this identification procedure is then given by

$$
L(q, \hat{\rho}, \hat{\eta})=\frac{D\left(q^{-1}, \hat{\eta}\right)}{F\left(q^{-1}, \hat{\rho}\right) C\left(q^{-1}, \hat{\eta}\right)} .
$$


- OE model structure:

When choosing an Output Error (OE) model structure for the noise dynamics, we arrive at

$$
y(t)=\frac{B\left(q^{-1}, \rho\right)}{F\left(q^{-1}, \rho\right)} u(t)+\varepsilon(t, \rho),
$$

and of course no additional estimation of the noise dynamics is required. The optimal prefilter however changes, and now becomes given by

$$
L(q, \hat{\rho})=\frac{1}{F\left(q^{-1}, \hat{\rho}\right)} .
$$

\subsection{Construction of the instruments}

According to (15), the instruments make use of the noise-free regressor $\stackrel{\varphi}{(t)}$ which is unknown. It has to be estimated, therefore, from data during the plant model estimation. Two situations have to be investigated according to the controller knowledge.

\subsubsection{Controller unknown}

When the controller is unknown, the IV series $\zeta_{f}(t, \hat{\theta})$ are generated on the basis of the two closedloop auxiliary models denoted as $G_{y r}\left(q, \hat{\rho}_{y r}\right)$ and $G_{u r}\left(q, \hat{\rho}_{u r}\right)$ (estimated TF between $y(t) / r(t)$ and $u(t) / r(t)$ respectively) as follows

$$
\begin{aligned}
\hat{y}(t) & =G_{y r}\left(q, \hat{\rho}_{y r}\right) r(t) \\
\hat{\grave{u}}(t) & =G_{u r}\left(q, \hat{\rho}_{u r}\right) r(t) \\
\zeta_{f}^{T}(t, \hat{\theta}) & =L(q, \hat{\theta})[-\hat{y}(t-1) \ldots-\hat{\grave{y}}(t-n) \hat{\dot{u}}(t-1) \ldots \hat{\dot{u}}(t-n)]
\end{aligned}
$$

where the closed-loop parameters $\hat{\rho}_{y r}$ and $\hat{\rho}_{u r}$ can be estimated by the following IV technique

$$
\begin{aligned}
& \hat{\rho}_{y r}=\left[\sum_{t=1}^{N} \zeta_{r}(t) \varphi_{y r}^{T}(t)\right]^{-1} \sum_{t=1}^{N} \zeta_{r}(t) y(t) \\
& \hat{\rho}_{u r}=\left[\sum_{t=1}^{N} \zeta_{r}(t) \varphi_{u r}^{T}(t)\right]^{-1} \sum_{t=1}^{N} \zeta_{r}(t) u(t)
\end{aligned}
$$

with the instruments $\zeta_{r}(t)$ generated from the excitation signal $r(t)$.

\subsubsection{Controller known}

When the controller is known, it is worthwhile to use this information into the identification procedure. Therefore, it may be used with the open-loop plant model to construct the instruments and the filter while satisfying the optimal conditions (13)-(15). Indeed, the two closed-loop 
transfer functions $\left(G_{y r}\left(q, \hat{\rho}_{y r}\right)\right.$ and $\left.G_{u r}\left(q, \hat{\rho}_{u r}\right)\right)$ are no longer needed and the noise-free estimation of the instrumental vector can be achieved by using the previous-step estimated open-loop transfer function. In this case, the IV series $\zeta_{f}(t, \hat{\theta})$ are generated on the following basis

$$
\begin{gathered}
\hat{\grave{y}}(t, \hat{\rho})=\frac{G(q, \hat{\rho})}{1+C_{c}(q) G(q, \hat{\rho})} r(t), \\
\hat{\dot{u}}(t, \hat{\rho})=\frac{1}{1+C_{c}(q) G(q, \hat{\rho})} r(t), \\
\zeta_{f}^{T}(t, \hat{\theta})=L(q, \hat{\theta})[-\hat{y}(t-1, \hat{\rho}) \ldots-\hat{y}(t-n, \hat{\rho}) \hat{\hat{u}}(t-1, \hat{\rho}) \ldots \hat{\dot{u}}(t-n, \hat{\rho})]
\end{gathered}
$$

\subsection{Algorithm}

The outline of the optimal IV algorithm for any model structures described Section 4.1, within the closed-loop context is given below.

\section{Step 1. Initialization}

- Estimate a first plant model, get $\hat{\rho}^{0}$ and denote $G\left(q, \hat{\rho}^{0}\right)=B\left(q^{-1}, \hat{\rho}^{0}\right) / F\left(q^{-1}, \hat{\rho}^{0}\right)$ the corresponding transfer function.

- Set the initial noise model estimates $C\left(q^{-1}, \hat{\eta}^{0}\right)=D\left(q^{-1}, \hat{\eta}^{0}\right)=1$ and $i=1$.

- If the controller is unknown, estimate as well the two closed-loop models (30)-(31) used in the following to build the instruments.

\section{Step 2. Estimate by IV}

Generate the filtered instruments ${ }^{3}$ according to the model structure used and the previous estimated models as

$$
\left\{\begin{array}{l}
L\left(q, \hat{\theta}^{i-1}\right) \text { computed using either }(18),(21),(24), \text { or }(26) \\
\zeta_{f}\left(t, \hat{\theta}^{i-1}\right)=L\left(q, \hat{\theta}^{i-1}\right)[-\hat{\grave{y}}(t-1) \ldots-\hat{\grave{y}}(t-n) \hat{\grave{u}}(t-1) \ldots \hat{\grave{u}}(t-n)]
\end{array}\right.
$$

where $\hat{\theta}^{i-1, T}=\left[\hat{\rho}^{i-1, T} \hat{\eta}^{i-1, T}\right]$, and $\zeta_{f}\left(t, \hat{\theta}^{i-1}\right)$ can be seen as a filtered estimate of the noise-free part of the regressor vector $\varphi(t)$ (9) based on estimates $\hat{y}(t)$ and $\hat{u}(t)$ of the noise-free output and input of the plant, respectively (using (27)-(28) if the controller is unknown and (32)-(33) if it is known). Determine the IV estimate using the prefilter and these instruments

$$
\begin{gathered}
\hat{\rho}^{i}=\left[\sum_{t=1}^{N} \zeta_{f}\left(t, \hat{\theta}^{i-1}\right) \varphi_{f}^{T}\left(t, \hat{\theta}^{i-1}\right)\right]^{-1} \sum_{t=1}^{N} \zeta_{f}\left(t, \hat{\theta}^{i-1}\right) y_{f}\left(t, \hat{\theta}^{i-1}\right) \\
\left\{\begin{array}{l}
\varphi_{f}\left(t, \hat{\theta}^{i-1}\right)=L\left(q, \hat{\theta}^{i-1}\right) \varphi(t) \\
y_{f}\left(t, \hat{\theta}^{i-1}\right)=L\left(q, \hat{\theta}^{i-1}\right) y(t)
\end{array}\right.
\end{gathered}
$$

\footnotetext{
${ }^{3} i$ stands for the ith iteration
} 
This yields $B\left(q^{-1}, \hat{\rho}^{i}\right)$ and $F\left(q^{-1}, \hat{\rho}^{i}\right)$. Denote the corresponding transfer function by $G\left(q, \hat{\rho}^{i}\right)=$ $B\left(q^{-1}, \hat{\rho}^{i}\right) / F\left(q^{-1}, \hat{\rho}^{i}\right)$.

Step 3. Obtain an estimate of the noise model parameter vector $\eta^{i}$ based on the estimated noise sequence

Use one of the noise model identification scheme ${ }^{4}$ described in Section 4 to estimate $\hat{\eta}^{i}$ and the associated transfer function $H\left(q, \hat{\eta}^{i}\right)$.

Step 4. Repeat from step 2. Stop when $F\left(q^{-1}, \hat{\rho}\right), B\left(q^{-1}, \hat{\rho}\right), H(q, \hat{\eta})$ and $L\left(q^{-1}, \hat{\theta}\right)$ have converged.

Step 5. Compute the estimated parametric error covariance matrix $\hat{P}_{\theta}$ associated with the parameter estimates, from

$$
\hat{P}_{\theta}=\hat{\sigma}^{2}\left[\varphi_{f}^{T}(t, \hat{\theta}) \varphi_{f}(t, \hat{\theta})\right]^{-1}
$$

where $\hat{\sigma}^{2}$ is the sample variance of the estimated residuals.

Then, according to the model structure used, the resulting algorithm will be referred to as cliv $_{a r x}$, cliv $_{\text {ararx }}$, cliv $_{b j}$ or clivee $_{\text {oe }}$

\section{Remark - Initialization step}

As illustrated in the following simulation example Section, the proposed optimal IV estimation method is robust to the initialization. Therefore, several methods may be used in this initialization step of the algorithm. Indeed, this first plant estimate is only used to construct the first filter and instruments, and therefore this suggests that minor deviations from the optimal value only causes second-order effects in the resulting accuracy of the first iteration model.

\section{Simulation examples}

These examples are concerned with a simulation model based on the following relations (1), where

$$
\begin{aligned}
G_{0}(q) & =\frac{0.0997 q^{-1}-0.0902 q^{-2}}{1-1.8858 q^{-1}+0.9048 q^{-2}}, \quad n=2 \\
C_{c}(q) & =\frac{10.75-9.25 q^{-1}}{1-q^{-1}},
\end{aligned}
$$

\footnotetext{
${ }^{4}$ One of the advantages of the proposed algorithms is that they provide consistent plant estimates while still exploiting the pseudo-linear regression type of estimation. Indeed, the IV based pseudo-linear regression method recently suggested in [21] could be used to estimate the ARMA process noise in this step of the algorithms.
} 
The excitation signal $r(t)$ is a pseudo random binary signal of maximal length, with the number of stages for the shift register set to 9 and the clock period set to $8 ; e_{0}(t)$ is a white noise uncorrelated with $r(t)$.

All of the IV methods presented in this paper lead to consistent results if the assumption $G_{0} \in \mathcal{G}$ is fulfilled and optimal estimates if $\mathcal{S} \in \mathcal{M}$. This example section illustrates this point with the use of the two following examples.

\subsection{Example 1: white measurement noise}

Firstly, a white noise disturbance $\left(H_{0}(q)=1\right)$ is considered in order to evaluate the performance of the cliv $_{o e}$ algorithm in the case $\mathcal{S} \in \mathcal{M}$. The plant parameters are estimated on the basis of closed-loop data of length $N=4088$. A Monte-Carlo simulation of 100 runs is used for a signal-to-noise (SNR) ratio given as

$$
\mathrm{SNR}=10 \log \left(\frac{P_{\grave{y}}}{P_{e}}\right)=35 \mathrm{~dB},
$$

where $P_{e}$ represents the average power of the zero-mean additive noise on the system output (e.g. the variance) while $P_{\grave{y}}$ denotes the average power of the noise-free output fluctuations. The proposed method is compared to:

- clivr: the first basic IV method developed to handle the closed-loop case which uses the delayed version of the reference signal as instruments [19];

- clivarx $_{\text {: }}$ the optimal IV method for an ARX model structure making use of the controller knowledge (see Section 4);

- cliv $_{o e 1}$ : the proposed IV method for OE model, assuming the controller unknown (see Section 4.3);

- cliv $_{o e 2}$ : the proposed IV method for OE model, making use of the controller knowledge (see Section 4.2.2);

- pem: direct use of the closed-loop data $(u(t)$ and $y(t))$ in the pem (OE here) method (see e.g. [1]). The method is known to be theoretically efficient in the $\mathcal{S} \in \mathcal{M}$ case.

Monte-Carlo simulation (MCS) results are presented in Table 1 where the mean and standard deviation of the estimated parameters are displayed. The average number of iterations $\left(N_{\text {iter }}\right)$ for the pem and cliv $_{o e}$ algorithms are also given. All the methods provide unbiased results with smaller standard deviations for the two proposed $c l i v_{o e}$ and pem methods. Indeed, these methods lead to better results thanks to the iterative estimation procedure, even though the number of iterations required for convergence is quite low (although it should be noted that, on 
average, pem requires more iterations to converge here). As expected, the clivr method provides the least accurate results since it is a basic (and not optimal) IV approach.

Moreover, it has to be noted that, contrary to the cliv $_{o e}$ and pem algorithms, the cliv $_{\text {arx }}$ method only works within the $G_{0} \in \mathcal{G}$ assumption (and not $\mathcal{S} \in \mathcal{M}$ ). However, even if the standard deviations are higher than those of the $c l i v_{o e}$ and pem, this refined IV provides satisfactory results and is robust to a miss-specified noise model.

Nevertheless, the proposed cliv $_{o e}$ algorithms allow to achieved more accurate results thanks to the appropriate noise model estimation. The two cliv $v_{o}$ methods (with or without the knowledge of the controller) perform similarly overall.

\begin{tabular}{|c|c|c|c|c|c|}
\hline $\begin{array}{c}\text { parameters } \\
\text { true values }\end{array}$ & $\hat{b}_{1}$ & $\hat{b}_{2}$ & $\hat{a}_{1}$ & $\hat{a}_{2}$ & $N_{\text {iter }}$ \\
\hline clivr & 0.0997 & -0.0902 & -1.8838 & 0.9048 & \\
cliv & $0.0997 \pm 0.30 e-3$ & $-0.0898 \pm 3.80 e-3$ & $-1.8821 \pm 35 e-3$ & $0.9014 \pm 32.4 e-3$ & \\
cliv $_{\text {oe } 1}$ & $0.0997 \pm 0.30 e-3$ & $-0.0900 \pm 1.10 e-3$ & $-1.8772 \pm 9 e-3$ & $0.8977 \pm 8.5 e-3$ & \\
cliv $_{\text {oe } 2}$ & $0.0997 \pm 0.05 e-3$ & $-0.0902 \pm 0.06 e-3$ & $-1.8858 \pm 0.12 e-3$ & $0.9048 \pm 0.10 e-3$ & 3.53 \\
pem & $0.0997 \pm 0.05 e-3$ & $-0.0902 \pm 0.06 e-3$ & $-1.8858 \pm 0.10 e-3$ & $0.9048 \pm 0.09 e-3$ & 3.89 \\
\hline
\end{tabular}

Table 1: Mean and standard deviation of the 100 estimated models, white measurement noise

\subsection{Example 2: colored measurement noise}

\subsection{1 $G_{0} \in \mathcal{G}$ case}

A second example is used to illustrate the performance of the proposed methods in the case of a colored measurement noise, with

$$
H_{0}(q)=\frac{1+0.5 q^{-1}}{1-0.85 q^{-1}}
$$

The following algorithms are used to estimate this model:

- the clivr method (with $G_{0} \in \mathcal{G}, \mathcal{S} \notin \mathcal{M}$ );

- the cliv $_{\text {ararx }}$ method (with $G_{0} \in \mathcal{G}, \mathcal{S} \notin \mathcal{M}$ );

- the $\operatorname{cliv}_{o e 1}$ method (with $G_{0} \in \mathcal{G}, \mathcal{S} \notin \mathcal{M}$ );

- cliv $_{b j 1}$ : the proposed IV method for BJ model, assuming the controller unknown (with $\mathcal{S} \in \mathcal{M})$

- $\operatorname{cliv}_{b j 2}$ : the proposed IV method for BJ model, making use of the controller knowledge $(\mathcal{S} \in \mathcal{M})$; 
- the pem algorithm (with $\mathcal{S} \in \mathcal{M})$.

The plant parameters are estimated on the basis of closed-loop data of length $N=4088$. A Monte-Carlo simulation of 100 runs is performed for SNR $=25 \mathrm{~dB}$. As previously, the results obtained using different algorithms are presented in Table 2 along with the average number of iterations needed for the cliv $_{b j}$ and pem algorithms. The Bode diagrams of the 100 models identified by the clivr, pem, cliv ararx and cliv $_{b j}$ algorithms are displayed in Figure 2.

As previously, all the IV-based methods give unbiased results. However, the basic clivr does clearly not deliver statistically efficient results. The additional noise model estimation in the refined IV type of algorithms allows to achieve statistically accurate results, especially with the cliv $_{b j}$ methods which makes it possible to exactly model the noise. Moreover, it could be noted that the clivarx is again robust to a miss-specified noise model. Furthermore, in Table 2 and Figure 2, it could be noticed that the pem algorithm (in the situation $\mathcal{S} \in \mathcal{M}$ ) is not able to converge to the global minimum at each run and therefore leads sometimes to erroneous results (see the large standard deviation of the pem estimates in Table 2). This is an expected result since it is well-known that this method may suffer from initialization issues (see e.g. [13] and [14]). However, better results may be obtained using the pem routine along with a more sophisticated multiple initialization step.

Additionally, it can be seen from Figure 2 that the proposed IV method named cliv $_{o e}$ still gives really accurate results (unbiased and low variance) even in the $G_{0} \in \mathcal{G}$ but $\mathcal{S} \notin \mathcal{M}$ case. Obviously, the variance is a little bit higher than with cliv $_{b j}$ but still satisfactory. This point illustrates the 'robustness' of the method face to a miss-specified noise model.

\begin{tabular}{|c|c|c|c|c|c|}
\hline $\begin{array}{c}\text { parameters } \\
\text { true values }\end{array}$ & $\hat{b}_{1}$ & $\hat{b}_{2}$ & $\hat{a}_{1}$ & $\hat{a}_{2}$ & $N_{\text {iter }}$ \\
\hline clivr & 0.0997 & -0.0902 & -1.8838 & 0.9048 & \\
cliv $_{\text {ararx }}$ & $0.0992 \pm 0.5 e-3$ & $-0.0900 \pm 4.9 e-3$ & $-1.8834 \pm 44.3 e-3$ & $0.9025 \pm 41.1 e-3$ & \\
cliv $_{b j}$ & $0.0997 \pm 0.6 e-3$ & $-0.0903 \pm 0.7 e-3$ & $-1.8860 \pm 3.7 e-3$ & $0.9050 \pm 3.4 e-3$ & 5 \\
cliv $_{b 2}$ & $0.0997 \pm 0.7 e-3$ & $-0.0903 \pm 0.7 e-3$ & $-1.8856 \pm 3.6 e-3$ & $0.9048 \pm 3.2 e-3$ & 5.88 \\
cliv $_{\text {oe } 1}$ & $0.0996 \pm 0.5 e-3$ & $-0.0901 \pm 0.7 e-3$ & $-1.8857 \pm 3.1 e-3$ & $0.9047 \pm 2.9 e-3$ & 5 \\
pem & $0.0793 \pm 64.8 e-3$ & $-0.0715 \pm 64 e-3$ & $-1.8993 \pm 34.7 e-3$ & $0.9181 \pm 33.8 e-3$ & 4.22 \\
\hline
\end{tabular}

Table 2: Mean and standard deviation of the 100 estimated models, colored measurement noise

\subsection{2 $\quad G_{0} \notin \mathcal{G}$ case}

This section illustrates the performance of the proposed method face to miss-specified process and noise model structures (with $\mathcal{S} \notin \mathcal{M}$ and $G_{0} \notin \mathcal{G}$ ). The example is the same as in Section 
5.2.1. A single experience is conducted using the cliv $_{o e}$ method, estimating a third order model and assuming a white noise model.

The results are given in Figure 3 where the comparison between the model output for the input signal and the measured output are depicted (over the first 1000 data points). Since we generated the data, we enjoy the luxury of comparing the model output to the noise-free system output (using the compare Matlab routine). The two plots coincide quite well and the coefficient of determination is equal to $98.9 \%$ (the pem routine on the same data set and assuming the same model structure leads to $81.4 \%$ ).

\section{Conclusion}

This paper has highlighted the use of optimal instrumental variable techniques in the closed-loop identification framework. Several available techniques have been presented in a new unified way according to the chosen model structure. Furthermore, a method able to estimate efficiently closed-loop Box-Jenkins models has been proposed. The general Box-Jenkins model structure identification has been handled by making use of a linear-in-the-parameters predictor next to an additional noise model identification required for determining the optimal filter and the instruments. The proposed method presents several advantages: it does not need to know the controller, it may be applied whatever the structure (linear or not) of the controller, it is able to handle the estimation of the more common use model structures (as ARX, ARARX, OE, BJ), it is quite robust to the initialization step and it does not suffer from convergence problem.

\section{References}

[1] U. Forssell and L. Ljung. Closed-loop identification revisited. Automatica, 35(7):1215-1241, 1999.

[2] M. Gevers. Towards a joint design of identification and control ?, chapter 5, pages 111151. H.L. Trentelman and J.C. Willems, Birkhäuser - Boston, 1993. Essay on Control : perspectives in the theory and its applications.

[3] M. Gevers. Identification for control: from the early achievements to the revival of experiment design. European Journal of Control, 11(4-5):335-352, 2005.

[4] M. Gilson, H. Garnier, P.C. Young, and P. Van den Hof. Instrumental variable methods for closed-loop continuous-time model identification. In Identification of Continuous-time Models from Sampled Data, pages 133-160. Springer, London, H. Garnier and L. Wang edition, 2008. 
[5] M. Gilson and P. Van den Hof. On the relation between a bias-eliminated least-squares (BELS) and an IV estimator in closed-loop identification. Automatica, 37(10):1593-1600, 2001.

[6] M. Gilson and P. Van den Hof. Instrumental variable methods for closed-loop system identification. Automatica, 41(2):241-249, 2005.

[7] G. Goodwin and J. Welsh. Bias issues in closed loop identification with application to adaptive control. Communications in Information and Systems, 2(4):349-370, December 2002.

[8] H. Hjalmarsson. From experiment design to closed-loop control. Automatica, 41(3):393-438, March 2005.

[9] T. Katayama. Subspace methods for system identification. Springer, 2005.

[10] I.D. Landau, A. Karimi, and A. Constantinescu. Direct controller order reduction by identification in closed loop. Automatica, 37(11):1689-1702, 2001.

[11] V. Laurain, M. Gilson, and H. Garnier. Refined instrumental variable methods for identifying hammerstein models operating in closed loop. In 48th IEEE Conference on Decision and Control (CDC'09), Shanghai - China, December 2009.

[12] L. Ljung. System Identification : Theory for the User - Second Edition. Prentice-Hall, 1999.

[13] L. Ljung. Initialisation aspects for subspace and output-error identification methods. In European Control Conference, Cambridge - UK, 2003.

[14] L. Ljung. Experiments with identification of continuous-time models. In 15th IFAC Symposium on System Identification, Saint Malo - France, June 2009.

[15] L. Mišković, A. Karimi, D. Bonvin, and M. Gevers. Closed-loop identification of multivariable systems: with or without excitation of all references? Automatica, 44(8):2048-2056, 2008.

[16] R. Pintelon and J. Schoukens. Box-jenkins identification revisited-part i: Theory. Automatica, 42(1):63-75, 2006.

[17] T. Söderström and P. Stoica. Instrumental Variable Methods for System Identification. Springer-Verlag, 1983.

[18] T. Söderström and P. Stoica. System identification. Prentice-Hall, 1989.

[19] T. Söderström, P. Stoica, and E. Trulsson. Instrumental variable methods for closed-loop systems. In 10th World IFAC Congress, pages 363-368, Munich - Germany, 1987. 
[20] P.M.J. Van den Hof. Closed-loop issues in system identification. Annual Reviews in Control, 22:173-186, 1998.

[21] P. Young. An instrumental variable approach to ARMA model identification and estimation. In 14th IFAC Symposium on System Identification, 2006.

[22] P.C. Young. An instrumental variable method for real-time identification of a noisy process. Automatica, 6:271-287, 1970.

[23] P.C. Young. Some observations on instrumental variable methods of time-series analysis. International Journal of Control, 23(5):593-612, 1976.

[24] P.C. Young. The refined instrumental variable method: unified estimation of discrete and continuous-time transfer function models. Journal Européen des Systèmes Automatisés, (42):149-179, 2008.

[25] P.C. Young and A. Jakeman. Refined instrumental variable methods of recursive time-series analysis - part i. single input, single output systems. International Journal of Control, 29(1):1-30, 1979.

[26] P.C. Young and A. Jakeman. Refined instrumental variable methods of recursive time-series analysis - part III. extensions. International Journal of Control, 31(4):741-764, 1980.

[27] W. X. Zheng. Application of BELS based methods in direct identification of linear systems from closed loop data. In 42nd IEEE Conference on Decision and Control, pages 4539-4544, Hawaï - USA, December 2003.

[28] W.X. Zheng. Parametric identification of linear systems operating under feedback control. IEEE Transactions on Circuits and Systems - I: Fundamental theory and applications, 48(4):451-458, 2001. 

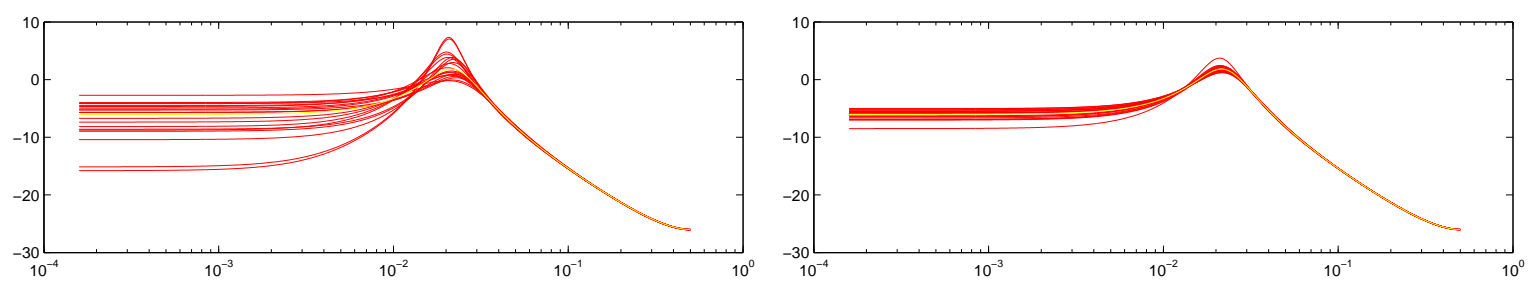

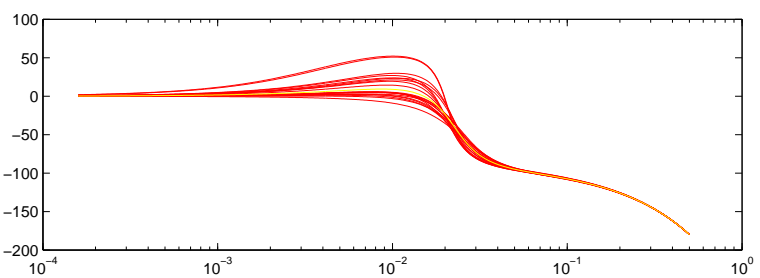

(a) clivr
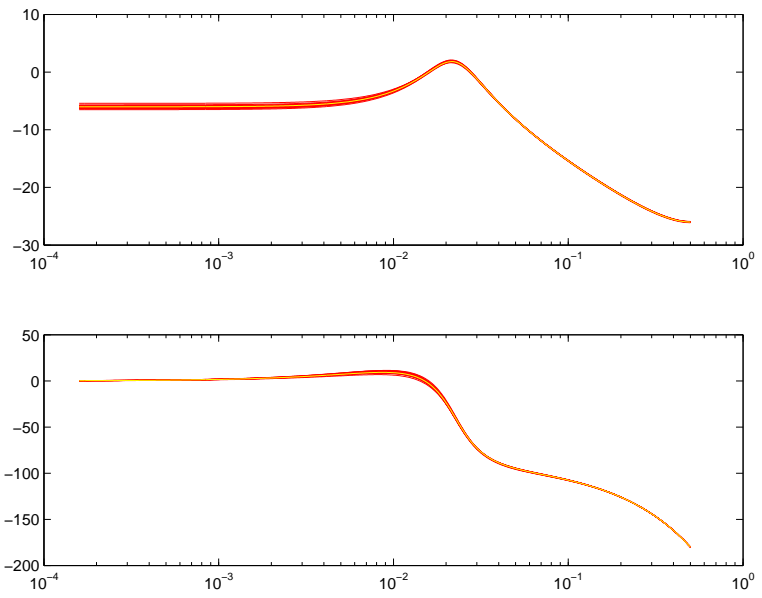

(c) $\operatorname{cliv}_{b j 1}$
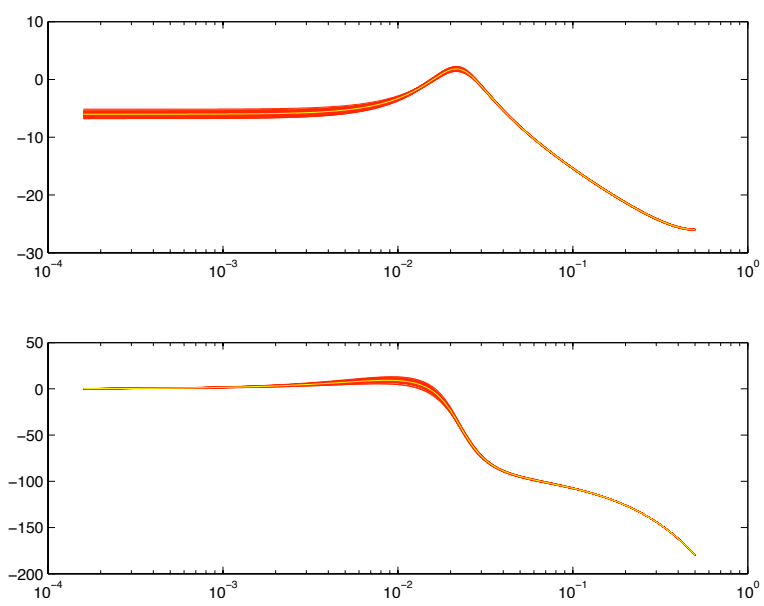

(e) $\operatorname{cliv}_{o e 1}$

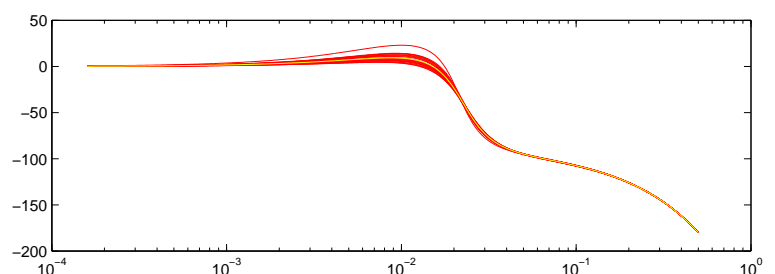

(b) cliv $_{\text {ararx }}$
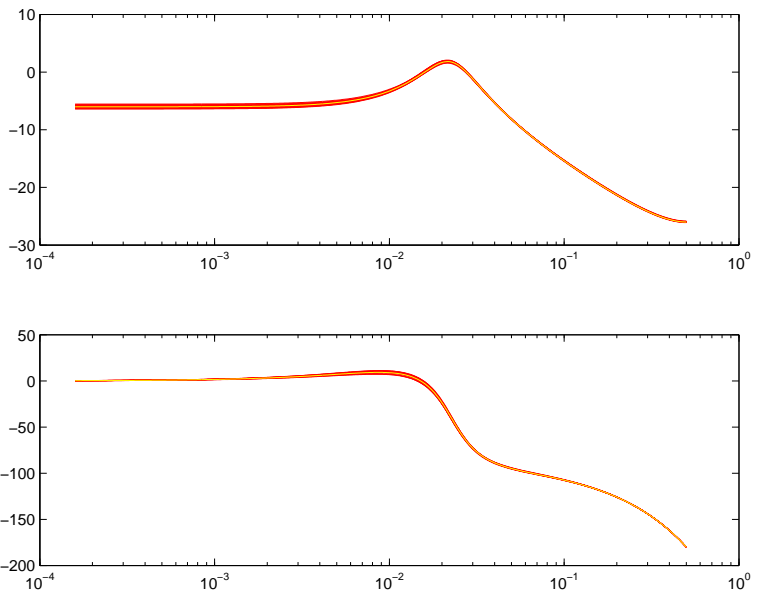

(d) $\operatorname{cliv}_{b j 2}$
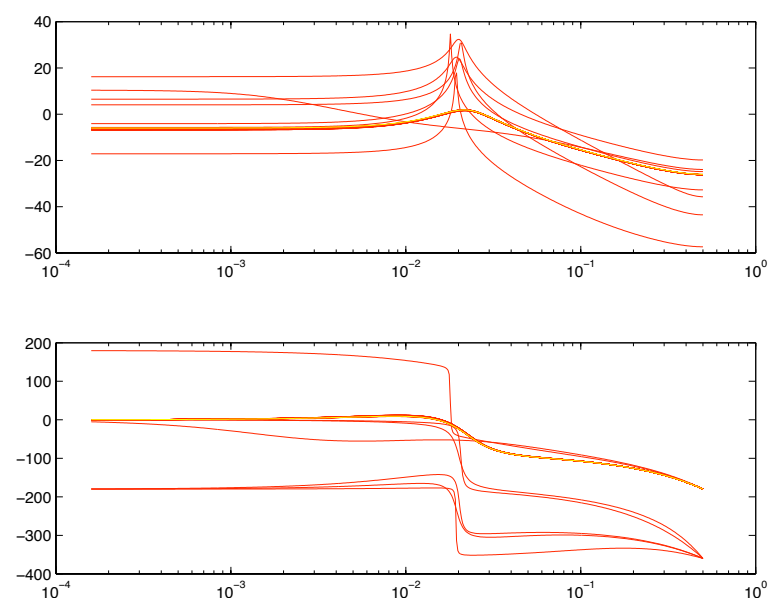

(f) pem

Figure 2: Estimated Bode diagrams (gain and phase (degree)) of the plant model $G(q, \eta)$ over the 100 MCS, colored noise 


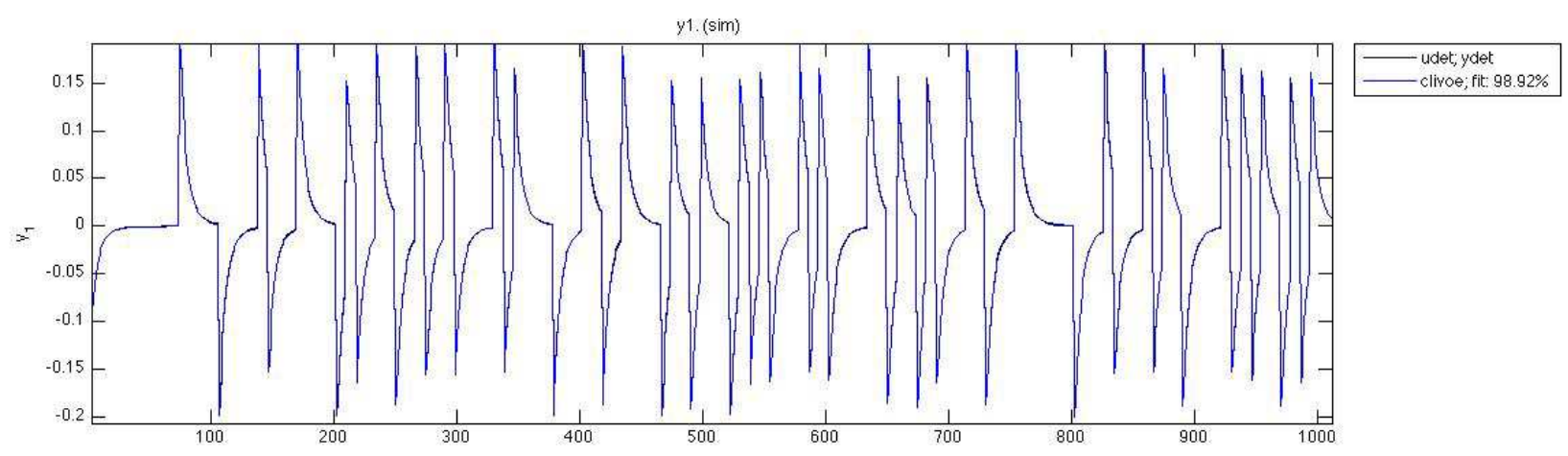

Figure 3: Estimated (with cliv $_{o e}$ ) and noise-free outputs in the $G_{0} \notin \mathcal{G}$ case 